\title{
钯催化烯烃杂环化反应制备[60]富勒烯二氢呋喃化合物
}

\author{
朱三娥* 豆礼锋 张建辉 吴缨 杨 伟 鲁红典 \\ 卫春祥 邓崇海董 强* \\ (合肥学院能源材料与化工学院 合肥 230601)
}

\begin{abstract}
摘要 富勒烯二氢呋喃衍生物在光电、医药等领域具有较好的应用前景, 近年来已成为研究热点. 以氯化钯为催化剂, 三氟甲烷磺酸铜为氧化剂, 烯烃衍生物和[60]富勒烯为原料, 通过 $\mathrm{C}-\mathrm{H}$ 键活化和杂环化反应, 合成了一系列单取代 和双取代的富勒烯二氢呋喃化合物. 利用 ${ }^{1} \mathrm{H} N \mathrm{NR} 、{ }^{13} \mathrm{C} N \mathrm{~N}$ 、 IR 和 HRMS 等对产物的结构进行了表征. 通过设计对 照实验, 对反应机理进行了研究, 提出了可能的反应机理.
\end{abstract}

关键词＼cjkstart烯烃; $\mathrm{C}_{60}$; 富勒烯二氢呋喃; 氯化钯; 三氟甲烷磺酸铜

\section{Palladium-Catalyzed Synthesis of Dihydrofuran-Fused [60]Fullerene Derivatives via Heteroannulation of Olefins}

\author{
Zhu, San'e* \\ Dou, Lifeng Zhang, Jianhui \\ Wu, Ying \\ Yang, Wei \\ Lu, Hongdian \\ Wei, Chunxiang \\ Deng, Chonghai \\ Dong, Qiang* \\ (School of Energy, Materials and Chemical Engineering, Hefei University, Hefei, Anhui 230601)
}

\begin{abstract}
With the good application prospects in the fields of optoelectronics and medicine, dihydrofuran-fused [60]fullerene derivatives have become a research hot topic in recent years. A series of monosubstituted and disubstituted dihydrofuran-fused [60]fullerene derivatives were synthesized by $\mathrm{C}-\mathrm{H}$ bond activation and heterocyclic reaction using palladium chloride as catalyst, copper trifluoromethanesulfonate as oxidant, olefins and [60]fullerene as raw materials. The structures of the products were confirmed by ${ }^{1} \mathrm{H}$ NMR, ${ }^{13} \mathrm{C}$ NMR, IR and HRMS. The reaction mechanism was studied by designing controlled experiments, and a possible reaction mechanism was proposed.

Keywords olefins; $\mathrm{C}_{60}$; dihydrofuran-fused [60]fullerene; palladium chloride; copper(II) trifluoromethanesulfonate
\end{abstract}

富勒烯由于具有特殊的几何结构和光电性能，被广 泛地应用于光电材料 ${ }^{[1-7]}$ 和光催化领域 ${ }^{[8-9]}$. 同时富勒烯 还具有优异的抗氧化能力 ${ }^{[10-11]}$ 、良好的细胞膜穿透能力 和调节离子传输能力 ${ }^{[12-13]}$, 因此有望被应用在高科技纳 米材料和医药工程领域 ${ }^{[14]}$. 与母体富勒烯相比, 在富勒 烯上引入活性官能团为其进一步应用于材料和医药领 域提供可能性 ${ }^{[15-17]}$.

二氢呋喃是一类重要的杂环化合物, 是很多药物和 天然化合物的重要结构单元 ${ }^{[18-19]}$, 广泛存在于抗肿

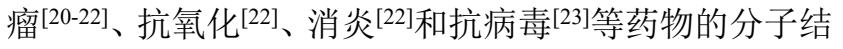
构中. 在富勒烯上引入二氢呋喃环, 有望开发出具有特
殊功能的纳米材料和医药中间体. 到目前为止，已报道 的富勒烯二氢呋喃类化合物的合成方法却非常有限.

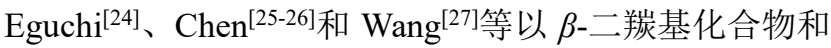
$\mathrm{C}_{60}$ 为原料在碱的催化下，以中等产率制备了双取代的 富勒烯二氢呋喃化合物. Wang ${ }^{[28-30]}$ 和 $\mathrm{Gao}^{[31]}$ 等以 1,3 -二 羰基化合物为原料在机械研磨或过渡金属如 $\mathrm{Ce}(\mathrm{IV})$ 、 $\mathrm{Mn}(\mathrm{III}) 、 \mathrm{Cu}(\mathrm{II}) 、 \mathrm{Fe}(\mathrm{III})$ 的体系中与 $\mathrm{C}_{60}$ 反应，合成了一 系列双取代的富勒烯二氢呋喃化合物. Liu 等利用 $\mathrm{Cu}(\mathrm{I}) / \mathrm{Ag}(\mathrm{I})$ 催化二芳基乙酮、苯甲酰乙腈及 $\beta$-二羰基化 合物与 $\mathrm{C}_{60}$ 反应，构筑了一系列双取代的富勒烯二氢呋 喃化合物 ${ }^{[32]}$.

\footnotetext{
* Corresponding authors. E-mail: 18096672005@163.com; qdong@hfuu.edu.cn

Received November 10, 2020; revised December 16, 2020; published online January 7, 2021.

Project supported by the National Natural Science Foundation of China (No. 21702042), the Program of Anhui Province for Outstanding Talents in University (Nos. gxyq2019114, gxyqZD2019085), and the Key Research and Development Project of Anhui Province (No. 201904b11020040).

国家自然科学基金(No. 21702042)、安徽省高校优秀人才(Nos. gxyq2019114, gxyqZD2019085)和安徽省重点研究与开发计划(No. 201904b11020040) 资助项目.
} 
上述制备富勒烯二氢呋喃类化合物的反应主要是 通过碳负离子的亲核加成与自由基相结合的反应机理 或者是过渡金属催化的自由基反应机理进行的，反应底 物一般是含有活泼亚甲基的化合物，如 $\beta$-二羰基化合 物、二芳基乙酮或苯甲酰乙腈等, 底物范围较窄, 且制 备的富勒烯二氢呋喃化合物是双取代的, 双键上都含有 两个取代基, 难以直接进行衍生化反应. 因此, 开发新 的反应底物和化学反应以制备结构新颖的富勒烯二氢 呋喃化合物具有非常重要的意义.

由于其原子经济性和环境友好等优点, 近几十年 来, 钯催化的 $\mathrm{C}-\mathrm{H}$ 键活化反应已经发展成一种高效构 筑 $\mathrm{C}-\mathrm{C}$ 键和 $\mathrm{C}-\mathrm{X}$ ( $\mathrm{X}$ 是杂原子)键的方法, 被广泛应用 于合成各种小分子化合物和天然产物 ${ }^{[33-36]}$. 自从 2009 年 Wang 课题组报道了钯催化的富勒烯与苯胺衍生物的 环化反应以来 ${ }^{[37]}$, 各种钯催化的富勒烯反应也被相继 报道 ${ }^{[38-40]}$. 但是利用钯催化直接制备富勒烯二氢呋喃类 化合物的研究报道比较少 ${ }^{[3,41-42]}$. 烯烃是重要的基础化 工原料, 也是重要的有机反应中间体, 不仅来源丰富, 而且易于衍生化. 但是到目前为止, 以烯烃为原料利用 钯催化直接制备富勒烯二氢呋喃化合物的反应却未见 报道. 因此, 本论文以 $\mathrm{C}_{60}$ 和烯烃衍生物为原料, 利用 $\mathrm{Pd}(\mathrm{II})$ 为催化剂, $\mathrm{Cu}(\mathrm{II})$ 为氧化剂, 通过优化反应条件构 筑了一系列富勒烯二氢呋喃化合物(如 Scheme 1 所示). 该方法不仅能够制备双取代的富勒烯二氢呋喃化合物, 而且可以构筑一系列单取代的富勒烯二氢呋喃化合物. 这些单取代的富勒烯二氢呋喃化合物中保留了原料烯 烃中的烯氢官能团, 有望直接进行衍生化反应, 以制备 具有特殊功能的新型纳米材料和医药中间体.

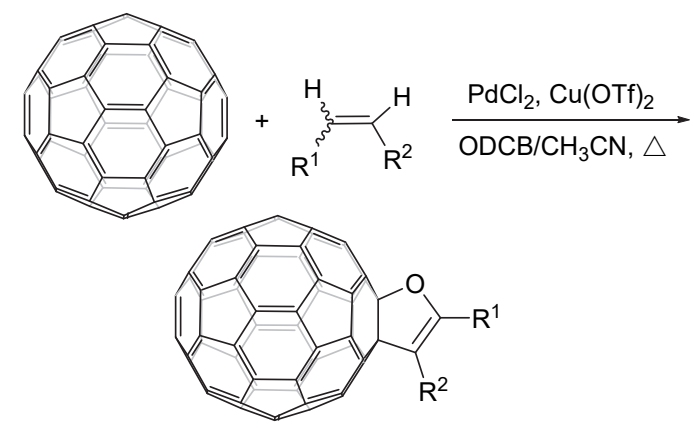

图式 1 由 $\mathrm{C}_{60}$ 和烯烃反应合成 $[60]$ 富勒烯二氢呋喃化合物 Scheme 1 Synthesis of dihydrofuran-fused [60]fullerene derivatives via $\mathrm{C}_{60}$ and olefins

\section{1 结果与讨论}

\section{1 反应条件的优化}

以丙烯酸乙酯(1a)为模板底物, 对反应条件进行优 化, 条件篎选如表 1 所示. 首先以 $\operatorname{Pd}(\mathrm{OAc})_{2}$ 为催化剂, 邻二氯苯和乙腈为溶剂篮选氧化剂. 当向反应体系中加
入常用的氧化剂如 $\mathrm{Cu}(\mathrm{OAc})_{2} 、\left(\mathrm{NH}_{4}\right)_{2} \mathrm{~S}_{2} \mathrm{O}_{8} 、 \mathrm{~K}_{2} \mathrm{~S}_{2} \mathrm{O}_{8}$ 、 Oxone 和 $\mathrm{Ag}_{2} \mathrm{CO}_{3}$ 时, 反应均不能发生(Entries 1 5). 利 用 $\mathrm{Cu}(\mathrm{TFA})_{2}$ 和 $\mathrm{Cu}(\mathrm{OTf})_{2}$ 作为氧化剂时反应可以进行, 均能得到预期产物, 其中以 $\mathrm{Cu}(\mathrm{OTf})_{2}$ 为氧化剂时反应效 果较好, 2a 的分离产率为 31\% (Entries 6 和 7). 因此, $\mathrm{Cu}(\mathrm{OTf})_{2}$ 被选择为该反应的氧化剂.

仅仅加入 $\mathrm{Cu}(\mathrm{OTf})_{2}$, 反应无预期产物生成(Entry 8). 说明钯在该反应体系中具有非常重要的作用. 于是以 $\mathrm{Cu}(\mathrm{OTf})_{2}$ 作为氧化剂, 对催化剂钯盐进行了篮选. 利用 钯盐 $\mathrm{Pd}(\mathrm{TFA})_{2} 、 \mathrm{Pd}\left(\mathrm{CH}_{3} \mathrm{CN}\right)_{4}(\mathrm{OTf})_{2}$ 和 $\mathrm{PdCl}_{2}$ 为催化剂时, 均能得到预期反应产物, 其中 $\mathrm{PdCl}_{2}$ 的催化效果最好, $2 \mathbf{a}$ 的分离产率提高到 38\% (Entries 9 11). 因此, $\mathrm{PdCl}_{2}$ 被 选择为该反应的催化剂.

随后还研究了酸碱对该反应的影响. 当向反应体系 中加入碱如 $\mathrm{NaOAc} \cdot 3 \mathrm{H}_{2} \mathrm{O} 、 \mathrm{NaHCO}_{3} 、 \mathrm{~K}_{2} \mathrm{CO}_{3}$ 和 $\mathrm{Na}_{2} \mathrm{CO}_{3}$, 碱对该环化反应并没有促进作用，反而会导致副产物的 生成和 2a 产率的降低(Entries 12 15). 酸如三氟醋酸 (TFA)或三氟甲烷磺酸的加入也没有得到更好的产率 (Entries 16 18). 可见, 在反应体系中无论是加入碱还 是酸, 对该反应均无促进作用.

最后, 研究了反应温度、氧化剂的用量、反应时间 和溶剂等对反应的影响. 降低反应温度、减少氧化剂的 用量、缩短反应时间均不利于该反应的进行 (Entries 19 21). 用 1,1,2,2-四氯乙烷/ $\mathrm{CH}_{3} \mathrm{CN}$ 为溶剂时, $\mathbf{2 a}$ 的分 离产率只有 $9 \%$ (Entry 22); 用纯邻二氯苯(ODCB)为溶 剂时, 可能是由于体系中的钯盐和铜盐不能够溶解, 反 应不能够发生(Entry 23).

综上所述, 合成化合物 $\mathbf{2 a}$ 的优化反应条件为: $\mathrm{C}_{60}$, 1a, $\mathrm{PdCl}_{2}$ 和 $\mathrm{Cu}(\mathrm{OTf})_{2}$ 的物质的量比为 $1: 5: 0.2: 5$, 反 应温度为 $130{ }^{\circ} \mathrm{C}$, 反应时间为 $10 \mathrm{~h}$, 反应溶剂为 $\mathrm{ODCB} /$ $\mathrm{CH}_{3} \mathrm{CN}(V: V=4: 1)$.

\section{2 反应底物的拓展}

为了探究该反应的底物适应性, 在上述优化反应条 件下研究了其它烯烃与 $\mathrm{C}_{60}$ 的反应, 结果如表 2 所示. 研 究发现, 在标准反应条件下 $\mathrm{R}^{1}$ 和 $\mathrm{R}^{2}$ 中必须有一个吸电 子基团, 反应才能够顺利进行. 首先研究了单取代烯烃 与 $\mathrm{C}_{60}$ 在标准体系中的反应. 除了丙烯酸乙酯(1a), 其它 丙烯酸酯如 $\mathbf{1 b}$ 和 $\mathbf{1 d}$ 也可以与 $\mathrm{C}_{60}$ 发生反应, 分别以 $29 \%$ 和 25\%的产率得到目标产物 $2 \mathbf{b}$ 和 2 d. 然而在相同 反应条件下, 丙烯酸叔丁酯 $(\mathbf{1} \mathbf{c})$ 与 $\mathrm{C}_{60}$ 的反应却不能够 进行, 造成这种反应活性差异的原因需要进一步研究. 除了脂肪族的酯, 丙烯酸苯酯(1e)也可以与 $\mathrm{C}_{60}$ 发生环 化反应, 以 $23 \%$ 的产率生成预期产物 2e. 随后研究了苯 环上取代基的电子效应对反应的影响. 当在丙烯酸苯酯 苯环的对位引入强给电子基团乙氧基(1f)和强吸电子 
表 1 合成化合物 $\mathbf{2 a}$ 的反应条件优化 ${ }^{a}$

Table 1 Optimization of the reaction conditions of compound $\mathbf{2 a}$
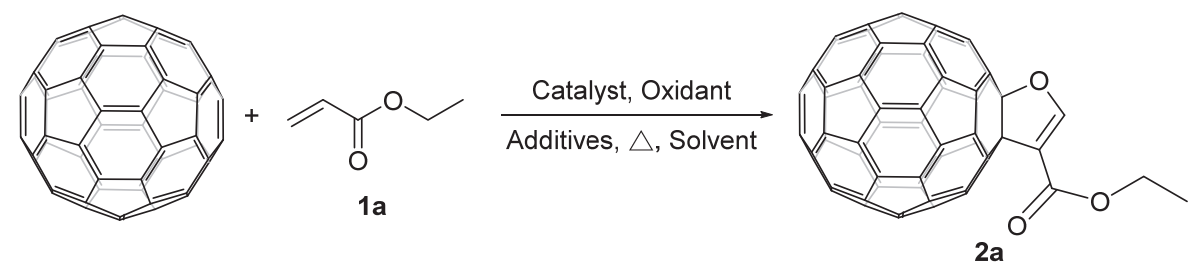

$2 \mathrm{a}$

\begin{tabular}{|c|c|c|c|c|c|}
\hline Entry & Catalyst & Oxidant & Base $^{b}$ & Acid $^{c}$ & Yield $/ \%$ \\
\hline 1 & $\mathrm{Pd}(\mathrm{OAc})_{2}$ & $\mathrm{Cu}(\mathrm{OAc})_{2}$ & - & - & 0 \\
\hline 2 & $\mathrm{Pd}(\mathrm{OAc})_{2}$ & $\left(\mathrm{NH}_{4}\right)_{2} \mathrm{~S}_{2} \mathrm{O}_{8}$ & - & - & 0 \\
\hline 3 & $\mathrm{Pd}(\mathrm{OAc})_{2}$ & $\mathrm{~K}_{2} \mathrm{~S}_{2} \mathrm{O}_{8}$ & - & - & 0 \\
\hline 4 & $\mathrm{Pd}(\mathrm{OAc})_{2}$ & Oxone & - & - & 0 \\
\hline 5 & $\mathrm{Pd}(\mathrm{OAc})_{2}$ & $\mathrm{Ag}_{2} \mathrm{CO}_{3}$ & - & - & 0 \\
\hline 6 & $\mathrm{Pd}(\mathrm{OAc})_{2}$ & $\mathrm{Cu}(\mathrm{TFA})_{2}$ & - & - & 15 \\
\hline 7 & $\mathrm{Pd}(\mathrm{OAc})_{2}$ & $\mathrm{Cu}(\mathrm{OTf})_{2}$ & - & - & 31 \\
\hline 8 & - & $\mathrm{Cu}(\mathrm{OTf})_{2}$ & - & - & 0 \\
\hline 9 & $\operatorname{Pd}(\mathrm{TFA})_{2}$ & $\mathrm{Cu}(\mathrm{OTf})_{2}$ & - & - & 23 \\
\hline 10 & $\mathrm{Pd}\left(\mathrm{CH}_{3} \mathrm{CN}\right)_{4}(\mathrm{OTf})_{2}$ & $\mathrm{Cu}(\mathrm{OTf})_{2}$ & - & - & 22 \\
\hline 11 & $\mathrm{PdCl}_{2}$ & $\mathrm{Cu}(\mathrm{OTf})_{2}$ & - & - & 38 \\
\hline 12 & $\mathrm{PdCl}_{2}$ & $\mathrm{Cu}(\mathrm{OTf})_{2}$ & $\mathrm{Na}_{2} \mathrm{CO}_{3}$ & - & 18 \\
\hline 13 & $\mathrm{PdCl}_{2}$ & $\mathrm{Cu}(\mathrm{OTf})_{2}$ & $\mathrm{~K}_{2} \mathrm{CO}_{3}$ & - & 29 \\
\hline 14 & $\mathrm{PdCl}_{2}$ & $\mathrm{Cu}(\mathrm{OTf})_{2}$ & $\mathrm{NaOAc} \cdot 3 \mathrm{H}_{2} \mathrm{O}$ & - & 23 \\
\hline 15 & $\mathrm{PdCl}_{2}$ & $\mathrm{Cu}(\mathrm{OTf})_{2}$ & $\mathrm{NaHCO}_{3}$ & - & trace \\
\hline 16 & $\operatorname{Pd}(\mathrm{TFA})_{2}$ & $\mathrm{Cu}(\mathrm{OAc})_{2}$ & - & TFA & trace \\
\hline 17 & $\mathrm{Pd}\left(\mathrm{CH}_{3} \mathrm{CN}\right)_{4}(\mathrm{OTf})_{2}$ & $\mathrm{Cu}(\mathrm{OAc})_{2}$ & - & $\mathrm{CF}_{3} \mathrm{SO}_{3} \mathrm{H}$ & 23 \\
\hline 18 & $\mathrm{PdCl}_{2}$ & $\mathrm{Cu}(\mathrm{OAc})_{2}$ & - & $\mathrm{CF}_{3} \mathrm{SO}_{3} \mathrm{H}$ & 33 \\
\hline $19^{e}$ & $\mathrm{PdCl}_{2}$ & $\mathrm{Cu}(\mathrm{OTf})_{2}$ & - & - & 30 \\
\hline $20^{f}$ & $\mathrm{PdCl}_{2}$ & $\mathrm{Cu}(\mathrm{OTf})_{2}$ & - & - & 11 \\
\hline $21^{g}$ & $\mathrm{PdCl}_{2}$ & $\mathrm{Cu}(\mathrm{OTf})_{2}$ & - & - & 31 \\
\hline $22^{h}$ & $\mathrm{PdCl}_{2}$ & $\mathrm{Cu}(\mathrm{OTf})_{2}$ & - & - & 9 \\
\hline $23^{i}$ & $\mathrm{PdCl}_{2}$ & $\mathrm{Cu}(\mathrm{OTf})_{2}$ & - & - & 0 \\
\hline
\end{tabular}

${ }^{a}$ Unless otherwise specified, all reactions were carried out with $\mathrm{C}_{60}(0.05 \mathrm{mmol}), 1 \mathrm{a}(0.25 \mathrm{mmol}), \mathrm{Pd}(\mathrm{II})(0.01 \mathrm{mmol})$, and an oxidant $(0.25 \mathrm{mmol})$ in $\mathrm{ODCB}(4$ $\mathrm{mL}) / \mathrm{CH}_{3} \mathrm{CN}(1 \mathrm{~mL})$ at $130{ }^{\circ} \mathrm{C}$ in air for $10 \mathrm{~h} .{ }^{b} 5$ eq. of base was used. ${ }^{c} 10$ eq. of acid was used. ${ }^{d}$ Isolated yield. ${ }^{e}$ The reaction was performed at $110{ }^{\circ} \mathrm{C} .{ }^{f} 2$ equiv. of $\mathrm{Cu}(\mathrm{OTf})_{2}$ was used. ${ }^{g}$ The reaction time was $5 \mathrm{~h} .{ }^{h} \mathrm{TCE}(4 \mathrm{~mL}) / \mathrm{CH}_{3} \mathrm{CN}(1 \mathrm{~mL})$ was used as solvent. ${ }^{I} \mathrm{ODCB}$ was used as solvent

表 2 烯烃底物的拓展 $a, b$

Table 2 Substrate scope of olefins
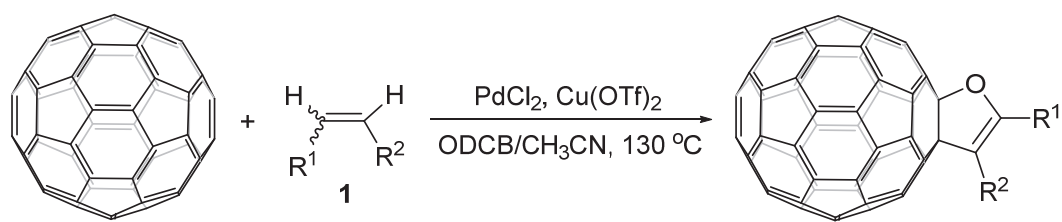

Substrate 1




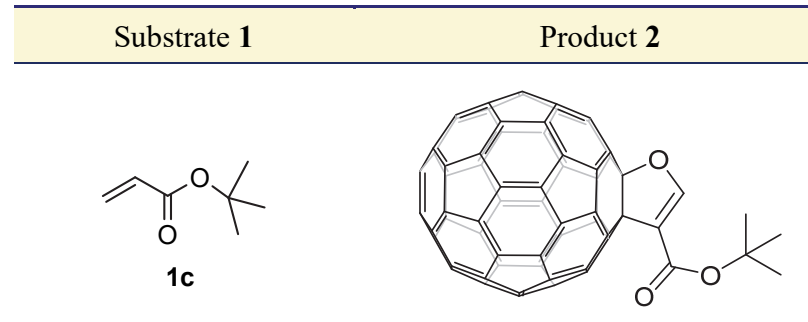

2c: $0 \%$<smiles>C=CC(=O)Oc1ccccc1</smiles>

$1 e$

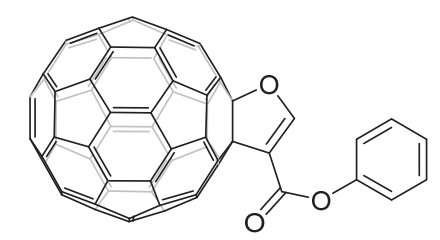

2e: $23 \%(82 \%)$<smiles>C=CC(=O)Oc1ccc([N+](=O)[O-])cc1</smiles>

$1 \mathrm{~g}$<smiles>C=CS(=O)(=O)c1ccccc1</smiles>

$1 \mathrm{i}$

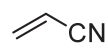

$1 \mathrm{k}$

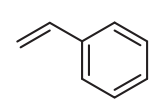

$1 \mathrm{~m}$

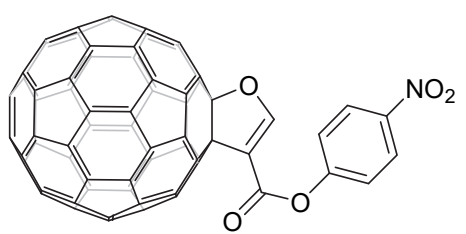

2g: $9 \%(86 \%)$

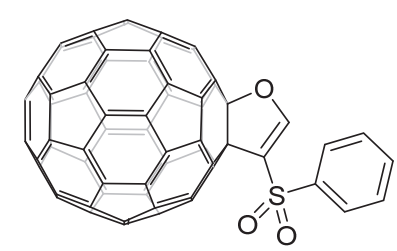

2i: $32 \%(89 \%)$

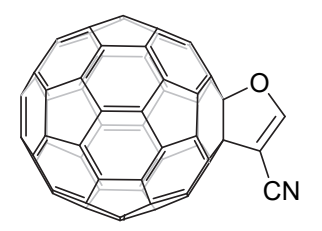

2k: $36 \%(87 \%)$

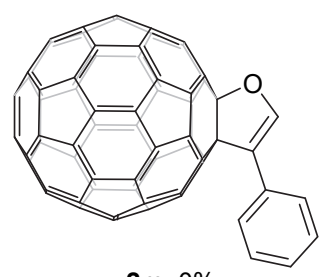

2m: 0\%<smiles>C=CC(=O)OCCOc1ccccc1</smiles>

1d

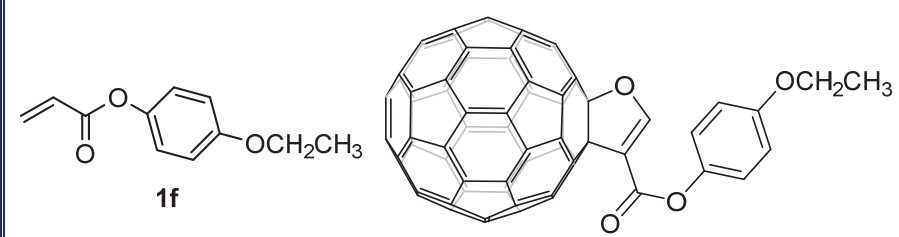

2f: $12 \%(82 \%)$<smiles>C=CP(=O)(OCC)OCC</smiles>

$1 \mathrm{~h}$

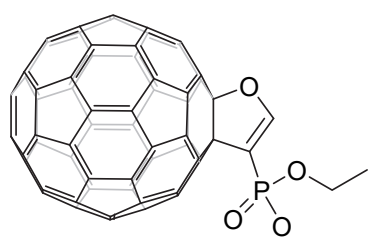

2h: 10\% (86\%)
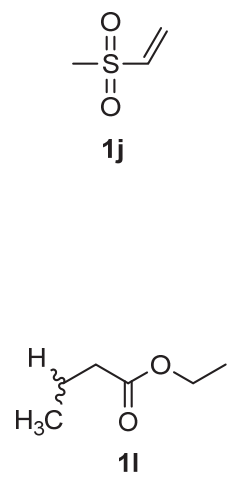

人ิ入

1n

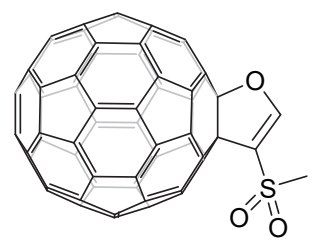

2j: 14\% (78\%)

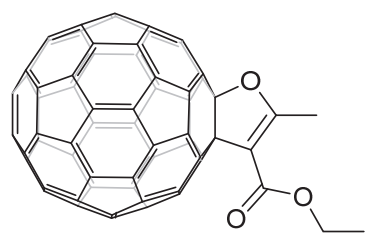

2I: $22 \%(79 \%)$

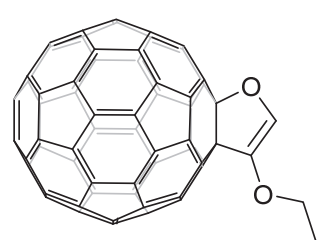

2n: $0 \%$

${ }^{a}$ Unless otherwise specified, all reactions were carried out with $\mathrm{C}_{60}(0.05 \mathrm{mmol}), \mathbf{1}(0.25 \mathrm{mmol}), \mathrm{PdCl}_{2}(0.01 \mathrm{mmol})$, and $\mathrm{Cu}(\mathrm{OTf})_{2}(0.25 \mathrm{mmol})$ in $\mathrm{ODCB}(4$ $\mathrm{mL}) / \mathrm{CH}_{3} \mathrm{CN}(1 \mathrm{~mL})$ at $130{ }^{\circ} \mathrm{C}$ in air for $10 \mathrm{~h} .{ }^{b}$ Isolated yield. Values in parentheses were based on consumed $\mathrm{C}_{60}$.

基团硝基 $(\mathbf{1 g})$ 时，反应也可以顺利发生，但是产物的分 离产率分别下降到 $12 \%$ 和 $9 \%$. 说明在苯环上引入强给 电子基团或强吸电子基团均不利于该反应的进行.

除了丙烯酸类型的底物, 还探究了其它单取代烯烃
衍生物如乙烯基磷酸酯、乙烯基砜和丙烯腈等与 $\mathrm{C}_{60}$ 的 反应. 在标准反应体系中, 乙烯基磷酸二乙酯(1h) 与 $\mathrm{C}_{60}$ 可以发生反应，以 $10 \%$ 的分离产率得到 $\mathbf{2 h}$. 苯基乙烯基 砜(1i)和甲基乙烯基砜(1j)也可以与 $\mathrm{C}_{60}$ 发生环化反应, 
分别以 $32 \%$ 和 $14 \%$ 的产率得到目标化合物 $\mathbf{2 i}$ 和 $\mathbf{2 j}$. 丙烯 腈在该反应体系中也可以顺利与 $\mathrm{C}_{60}$ 反应，以 $36 \%$ 的产 率得到目标化合物 $\mathbf{2 k}$.

此外, 双取代烯烃如巴豆酸乙酯 11 , 在该反应体系 中也可以与 $\mathrm{C}_{60}$ 顺利发生环化反应, 以 $22 \%$ 的产率生成 目标化合物 21.

最后, 还研究了富电子烯烃苯乙烯 $\mathbf{1 m}$ 和乙基乙烯 基醚 $1 \mathbf{n}$ 与 $\mathrm{C}_{60}$ 的反应. 很遗憾, 在标准反应体系中, 均 未得到预期产物.

\section{3 化合物的结构分析}

由烯烃衍生物 $\mathbf{1}$ 与 $\mathrm{C}_{60}$ 通过环化反应制备富勒烯二 氢呋喃化合物, 从理论上来说, 可以生成如 Scheme 2 所 示的 2 和 2'两种结构. 为了分析在该反应体系中得到的 富勒烯二氢呋喃的结构, 进而探究该反应的机理, 又开 展了相应的对照实验.

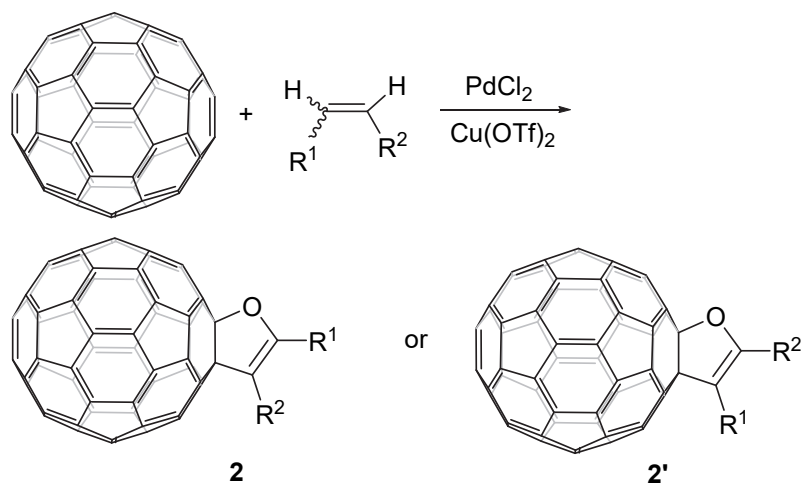

图式 2 由 $\mathrm{C}_{60}$ 和烯烃反应制备的 [60]富勒烯二氢呋喃化合物 可能存在的结构

Scheme 2 Possible structures of dihydrofuran-fused [60]fullerene derivatives synthesized via olefins and $\mathrm{C}_{60}$

在以上反应体系中, 合成的富勒烯二氢呋喃化合物 多数都是单取代的, 而目前所报道的富勒烯二氢呋喃化 合物大都是双取代的，因此，很难直接通过与文献对比 确定产物的结构是 2 还是 $\mathbf{2}^{\prime}$. 当利用巴豆酸乙酯与 $\mathrm{C}_{60}$ 进行环化反应时, 理论上可以生成双取代富勒烯二氢呋 喃化合物 21 或者 2I'，具体结构如图 1 所示. 如果生成的 产物结构是 21, 那么该化合物的结构是已经报道 的 ${ }^{[24,27,29-30]}$, 可以通过结构对比来确定.

如果在相同的测试条件下, 巴豆酸乙酯与 $\mathrm{C}_{60}$ 反应 的产物与 21 的结构表征数据相同, 那么说明该产物的结 构是 21. 为了在相同的测试条件下进行对比, 首先需要 制备化合物 21. 于是参照文献报道的方法, 在醋酸铜和 DMAP 的体系中，以乙酰乙酸乙酯和 $\mathrm{C}_{60}$ 为原料合成了 已知化合物 2[ ${ }^{[29]}$, 反应如 Scheme 3 所示. 随后, 分别对 巴豆酸乙酯与 $\mathrm{C}_{60}$ 反应的产物和对照实验中合成的 21

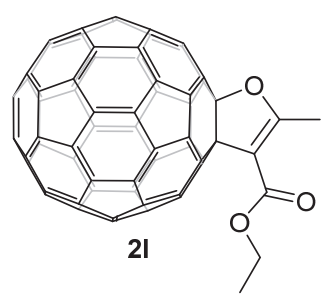

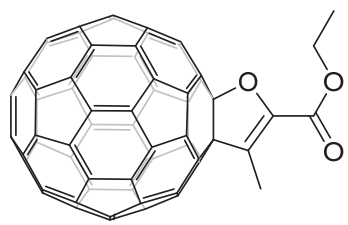

2l'
图 121 和 2I'的结构

Figure 1 The structures of $\mathbf{2 I}$ and $\mathbf{2} \mathbf{I}^{\prime}$

进行了氢谱和碳谱表征. 通过分析发现，这两个化合物 的 ${ }^{1} \mathrm{H}$ NMR 完全一致, 均在 $\delta 4.08$ 处出现对应于 $\mathrm{OCH}_{2} \mathrm{CH}_{3}$ 的四重峰, 在 $\delta 0.998$ 处出现对应于 $\mathrm{OCH}_{2} \mathrm{CH}_{3}$ 的三重峰, 在 $\delta 2.65$ 处出现对应于 $\mathrm{CH}_{3}$ 的单峰; 在 ${ }^{13} \mathrm{C}$ NMR 谱图上, 除了溶剂比例导致的微小化学位移差别 外，这两个化合物的化学位移也完全一致. 由此可以确 定，在标准反应体系中，巴豆酸乙酯与 $\mathrm{C}_{60}$ 反应生成的 产物结构是 $2 \mathbf{2 l}$, 而不是 $2 \mathbf{l}^{\prime}$.

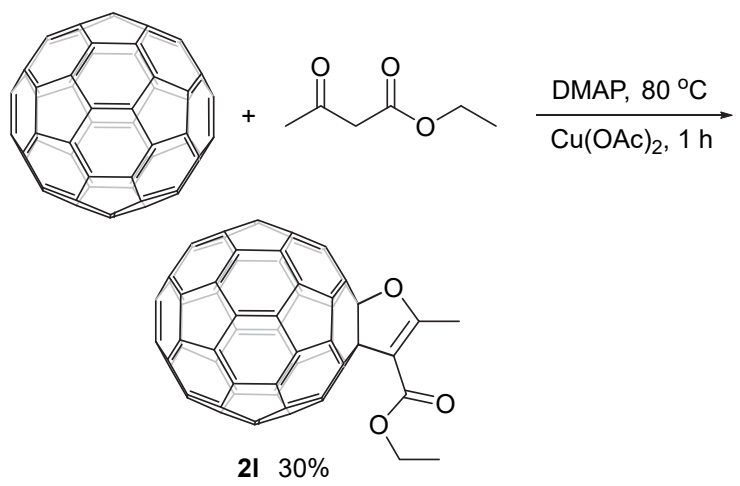

图式 3 对照实验: $\mathrm{C}_{60}$ 和乙酰乙酸乙酯反应合成 21 Scheme 3 Control experiment: synthesis of compound $\mathbf{2 l}$ via $\mathrm{C}_{60}$ and ethyl acetoacetate

同样地，在该反应体系中，其它烯烃衍生物与 $\mathrm{C}_{60}$ 反应生成的产物结构应该是 2 , 而不是 $\mathbf{2}^{\prime}$. 为了确认产 物的结构, 利用核磁共振、高分辨质谱和红外光谱等分 析手段对各化合物的结构进行了表征. 化合物 $\mathbf{2 a} \sim 21$ 的 高分辨质谱均给出相应的负离子自由基峰. 在 ${ }^{1} \mathrm{H}$ NMR 上, 所有单取代的富勒烯二氢呋喃化合物的化学位移 $\delta$ 在 $7.8 \sim 8.7$ 区间均有一个单峰出现, 对应于二氢呋喃环 上的烯氢. 在 ${ }^{13} \mathrm{C}$ NMR 上, 在 $\delta \quad 157 \sim 159$ 和 $111 \sim 121$ 处出现的峰对应于二氢呋喃环双键上的两个碳; 所有化 合物在 $\delta 135 \sim 148$ 区间均出现多条谱线，其数量不超过 30 条, 对应于 $\mathrm{C}_{60}$ 笼上 $\mathrm{sp}^{2}$ 碳原子的信号, 与化合物的 $C_{s}$ 对称性一致; $\mathrm{C}_{60}$ 的两个 $\mathrm{sp}^{3}$ 杂化的碳原子信号分别出 现在 $\delta 69 \sim 72$ 和 105 109 区间. 这些都与文献上已经 报道的富勒烯二氢呋喃类化合物的谱图类似 [24,27,29-31], 因此可以确定化合物 $\mathbf{2 a} \sim 2 \mathbf{k}$ 的结构. 


\section{2 反应机理研究}

与原料 $\mathrm{C}_{60}$ 和烯烃衍生物相比较, 产物 $\mathbf{2 a} \sim \mathbf{2 1}$ 的结 构中多了一个氧原子. 以 $1 \mathrm{i}$ 到 $2 \mathrm{i}$ 的反应为模板 (1i 为固 体原料, 可以避免在无水无氧操作中发生损失), 通过 开展对照实验, 探究氧原子的来源, 对可能的反应机理 进行了研究.

首先考虑产物中的氧原子是不是来自于空气中的 氧. 为此, 以 $1 \mathbf{i}$ 为原料, 设计了(i)、(ii)和(iii)三组对照实 验，具体如 Scheme 4 所示. (i) 是严格无氧条件下的反应; (ii)是在通氧气条件下的反应; (iii)是在标准条件下的反 应. 研究发现, 三组反应均能够顺利进行, 且 $2 \mathbf{i}$ 的分离 产率几乎没有变化. 说明产物中的氧并不是来自于空气 中的氧气.

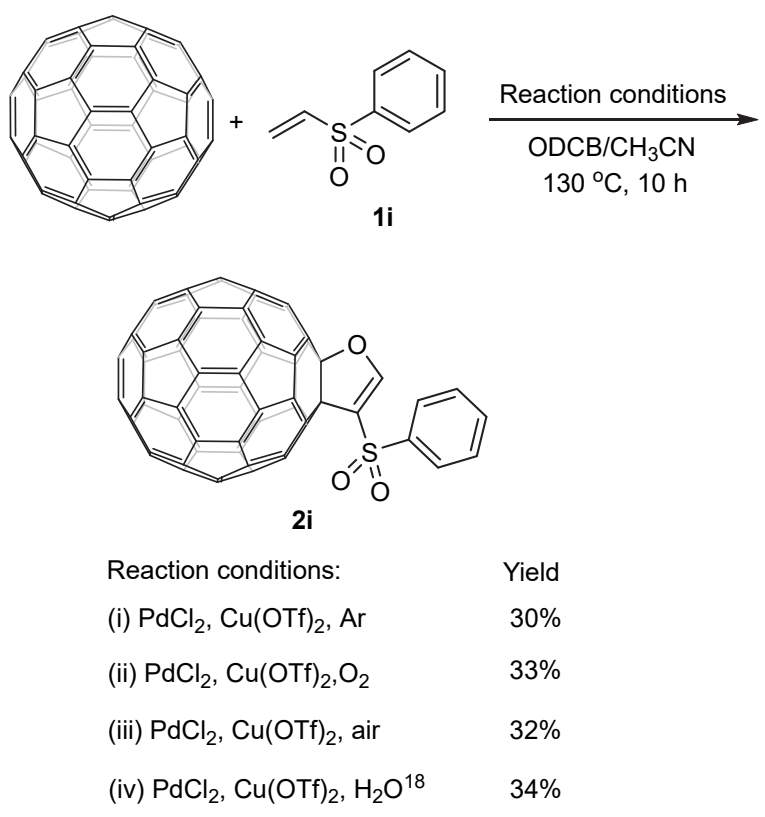

图式 4 反应机理的探究

Scheme 4 Probe reaction mechanism

其次, 考虑产物中的氧是不是来源于水. 如 Scheme 4 的反应(iv)所示, 在标准体系中加入 $20 \mu \mathrm{L}$ 的 $\mathrm{H}_{2} \mathrm{O}^{18}$, 封 管反应 $10 \mathrm{~h}$ 后, 得到与 $\mathbf{2} \mathbf{i}$ 极性相同的产物. 对产物进行 高分辨质谱表征发现, 产物的负离子自由基的质量 $\left[\mathrm{M}^{\prime^{-}}\right.$]为 904.0082 (对应于 $\mathrm{C}_{68} \mathrm{H}_{6} \mathrm{O}_{2} \mathrm{O}^{18} \mathrm{~S}^{\cdot-}$ ), 比 $2 \mathbf{i}$ 的负离 子自由基的质量(902.0048)多 2. 说明产物中的氧是来源 于体系中的 $\mathrm{H}_{2} \mathrm{O}$.

结合已有文献, 推测可能的反应机理如 Scheme 5 所示. 首先, 在钯的催化下, 水与烯烃发生亲核加成反 应，再经过消除反应生成烯醇 $\mathbf{D}^{[43-46]}$; 然后通过 $\mathrm{C}-\mathrm{H}$ 活化, 与富勒烯形成六元钯环中间体 $\mathbf{F}^{[41-42]}$; 再经过还 原消除, 形成产物 2 和 $\operatorname{Pd}(0)$; 最后 $\operatorname{Pd}(0)$ 再被 $\mathrm{Cu}(\mathrm{II})$ 氧 化, 再生 Pd(II) ${ }^{[42]}$.

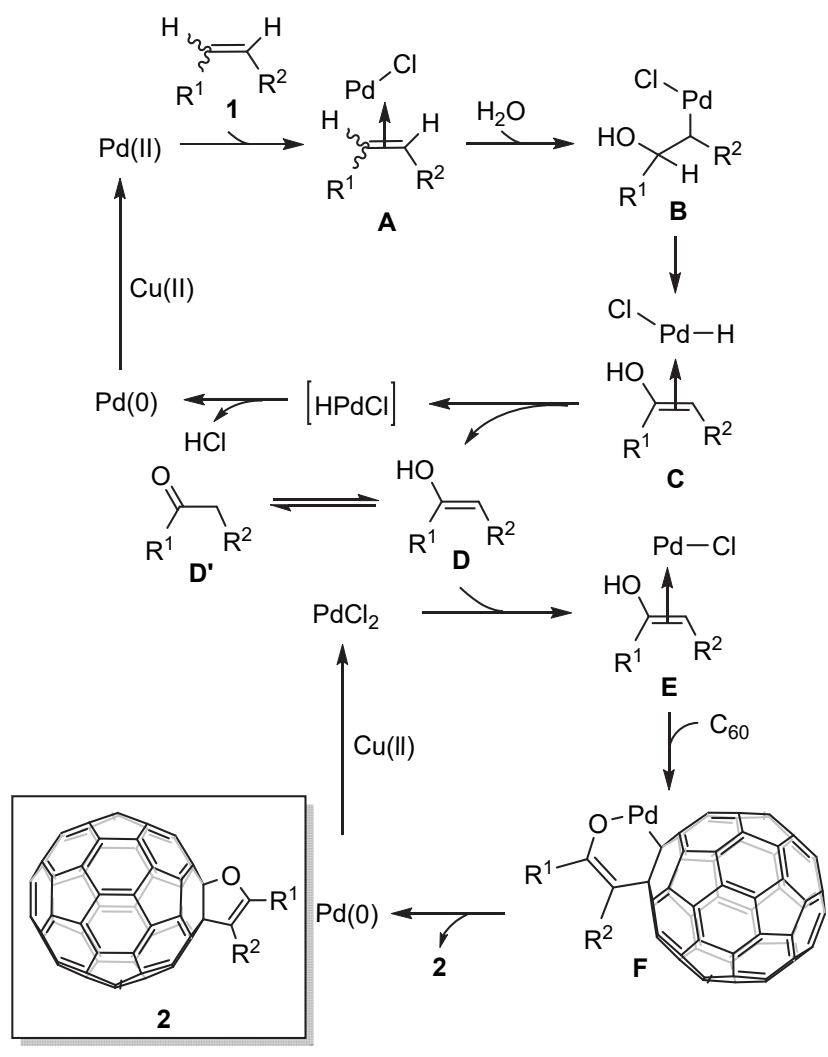

图式 5 可能的反应机理

Scheme 5 Proposed reaction mechanism

为了验证烯烃是先生成烯醇再与 $\mathrm{C}_{60}$ 反应的过程, 又用乙酰乙酸乙酯代替巴豆酸乙酯 11 在标准条件下进 行反应. 结果表明, 乙酰乙酸乙酯在该反应条件下也可 以顺利进行反应, 得到 21 , 进一步验证了该反应机理的 可行性.

\section{3 结论}

以烯烃衍生物为原料, 利用 $\mathrm{PdCl}_{2}$ 为催化剂, $\mathrm{Cu}(\mathrm{OTf})_{2}$ 为氧化剂, 通过 $\mathrm{C}-\mathrm{H}$ 活化和杂环化反应合成 了一系列单取代和双取代的富勒烯二氢呋喃化合物, 并 通过设计对照实验，提出了可能的反应机理. 该方法中 原料烯烃来源丰富, 而且得到的单取代富勒烯二氢呋喃 化合物中保留了活性官能团烯氢, 为其直接功能化反应 以制备具有特殊功能的纳米材料和医药中间体奠定基 础.

\section{4 实验部分}

\section{1 仪器与试剂}

${ }^{1} \mathrm{H}$ NMR 和 ${ }^{13} \mathrm{C}$ NMR 由 Bruker AM-400 (400 MHz) 型核磁共振仪测定 $\left(\mathrm{CDCl}_{3}-\mathrm{CS}_{2}, \mathrm{CS}_{2} / \mathrm{DMSO}-d_{6}\right.$ 或 $o$-Dichlorobenzene- $d_{4}$ 作溶剂, TMS 作内标); IR 由美国 Thermo Fisher 公司的 Nicolet iS $50+$ 傅立叶变换红外光 
谱仪测定; HRMS 使用 Bruker ultraflextreme MALDI TOF/TOF 在负离子模式下测定. 溶剂邻二氯苯和乙腈 使用无水级别. 其它所有药品和试剂均为商品化的分析 纯或化学纯.

\section{2 实验方法}

\subsection{1 化合物 $\mathbf{2 a} \sim 2 \mathbf{2}$ 的合成}

在装有搅拌子的 $15 \mathrm{~mL}$ 的封管中, 加入 $\mathrm{C}_{60}(36.0$ $\mathrm{mg}, 0.05 \mathrm{mmol})$, 烯烃衍生物 1 ( $0.25 \mathrm{mmol}), \mathrm{PdCl}_{2}$ (1.8 $\mathrm{mg}, 0.01 \mathrm{mmol}$ )和 $\mathrm{Cu}(\mathrm{OTf})_{2}(90.4 \mathrm{mg}, 0.25 \mathrm{mmol})$; 然后 再加入 $4 \mathrm{~mL}$ 的邻二氯苯和 $1 \mathrm{~mL}$ 的乙腈. 将封管密封后, 超声溶解, 并置于 $130{ }^{\circ} \mathrm{C}$ 的油浴锅中反应 $10 \mathrm{~h}$. 反应完 成后, 将反应液冷却至室温. 减压蒸馏除去溶剂邻二氯 苯和乙腈, 用柱色谱进行分离. 首先用二硫化碳作为淋 洗剂得到未反应的 $\mathrm{C}_{60}$ (回收 $\mathrm{C}_{60}$ ), 然后根据产物极性, 用二硫化碳/二氯甲烷作为淋洗剂, 柱层析分离得到目 标化合物 2.

[60]富勒烯稠合-4,5-二氢-3-呋喃甲酸乙酯(2a): 棕 黑色固体 $15.9 \mathrm{mg}$, 产率 38\%(回收 $\mathrm{C}_{60} 19.7 \mathrm{mg}$ ). m.p.> $300{ }^{\circ} \mathrm{C}$; UV-vis $\left(\mathrm{CHCl}_{3}\right) \lambda_{\max }: 256,316,426 \mathrm{~nm} ;{ }^{1} \mathrm{H}$ NMR (400 MHz, $\left.\mathrm{CS}_{2}-\mathrm{CDCl}_{3}\right) \delta: 8.05$ (s, 1H), $4.13(\mathrm{q}, J=6.9 \mathrm{~Hz}$, $2 \mathrm{H}), 1.10(\mathrm{t}, J=7.0 \mathrm{~Hz}, 3 \mathrm{H}) ;{ }^{13} \mathrm{C}$ NMR $(100 \mathrm{MHz}$, $\left.\mathrm{CS}_{2}-\mathrm{CDCl}_{3}\right) \delta: 163.09,157.01,147.98,147.58,147.20$, $146.98,146.38,146.21,146.07,145.94,145.91,145.50$, $145.41,145.12,144.98,144.62,144.43,144.08,143.50$, $142.74,142.71,142.64,142.43,142.37,142.26,142.22$, $141.59,141.38,139.82,139.79,137.47,135.30,111.34$, 105.58, 70.24, 60.68, 14.57; FT-IR (KBr) v: 2957, 2923, 2852, 1706, 1622, 1506, 1462, 1367, 1321, 1262, 1216, $1171,1155,1122,1092,940,898,748,692,525 \mathrm{~cm}^{-1}$. HRMS (MALDI-TOF) calcd for $\mathrm{C}_{65} \mathrm{H}_{6} \mathrm{O}_{3} 834.0322$, found 834.0311

[60]富勒烯稠合-4,5-二氢-3-呋喃甲酸正丁酯(2b): 棕黑色固体 $12.5 \mathrm{mg}$, 产率 29\%(回收 $\mathrm{C}_{60} 23.9 \mathrm{mg}$ ). m.p. $>300{ }^{\circ} \mathrm{C}$; UV-vis $\left(\mathrm{CHCl}_{3}\right) \lambda_{\max }: 258,314,427 \mathrm{~nm} ;{ }^{1} \mathrm{H}$ NMR (400 MHz, $\left.\mathrm{CS}_{2}-\mathrm{CDCl}_{3}\right) \delta: 8.27(\mathrm{~s}, 1 \mathrm{H}), 4.28(\mathrm{t}, J=$ $6.6 \mathrm{~Hz}, 2 \mathrm{H}), 1.75 \sim 1.68(\mathrm{~m}, 2 \mathrm{H}), 1.45(\mathrm{dt}, J=7.4 \mathrm{~Hz}, 2 \mathrm{H})$, 0.98 (t, $J=7.4 \mathrm{~Hz}, 3 \mathrm{H}) ;{ }^{13} \mathrm{C}$ NMR $\left(100 \mathrm{MHz}, \mathrm{CS}_{2}-\mathrm{CDCl}_{3}\right)$ $\delta: 163.19,157.06,147.99,147.58,147.21,146.96,146.38$, $146.22,146.08,145.95,145.91,145.51,145.41,145.13$, $144.99,144.62,144.43,144.09,143.51,142.75,142.72$, $142.64,142.44,142.37,142.26,142.23,141.58,141.39$, $139.83,139.78,137.48,135.32,111.43,105.59,70.24$, 64.56, 31.04, 19.73, 14.04; FT-IR (KBr) v: 2953, 2923, $2859,1705,1625,1513,1461,1377,1321,1277,1217$, $1155,1123,1093,941,898,577,526 \mathrm{~cm}^{-1}$. HRMS
(MALDI-TOF) calcd for $\mathrm{C}_{67} \mathrm{H}_{10} \mathrm{O}_{3}$ 862.0635, found 862.0619 .

[60]富勒烯稠合-4,5-二氢-3-呋喃甲酸苯氧基乙酯 (2d): 棕黑色固体 $11.6 \mathrm{mg}$, 产率 25\%(回收 $\mathrm{C}_{60} 25.9 \mathrm{mg}$ ). m.p. $>300{ }^{\circ} \mathrm{C}$; UV-vis $\left(\mathrm{CHCl}_{3}\right) \lambda_{\max }: 255,315,423 \mathrm{~nm} ;{ }^{1} \mathrm{H}$ NMR (400 MHz, $\left.\mathrm{CS}_{2}-\mathrm{CDCl}_{3}\right) \delta: 8.32(\mathrm{~s}, 1 \mathrm{H}), 7.24(\mathrm{~d}, J=$ $7.4 \mathrm{~Hz}, 2 \mathrm{H}), 6.91(\mathrm{t}, J=7.3 \mathrm{~Hz}, 1 \mathrm{H}), 6.82(\mathrm{~d}, \quad J=7.8 \mathrm{~Hz}$, $2 \mathrm{H}), 4.63(\mathrm{t}, J=4.6 \mathrm{~Hz}, 2 \mathrm{H}), 4.22(\mathrm{t}, J=4.6 \mathrm{~Hz}, 2 \mathrm{H}) ;{ }^{13} \mathrm{C}$ NMR $\left(100 \mathrm{MHz}, \mathrm{CS}_{2}-\mathrm{CDCl}_{3}\right) \delta: 162.94,158.10,157.88$, $147.92,147.36,147.15,146.76,146.33,146.17,146.01$, $145.89,145.85,145.43,145.34,145.06,144.93,144.55$, $144.35,144.03,143.32,142.67,142.62,142.58,142.33$, $142.29,142.20,142.17,141.46,141.31,139.77,139.73$, $137.46,135.27,129.45,121.19,114.35,111.01,105.71$, 70.03, 65.51, 63.08; FT-IR (KBr) v: 2926, 1708, 1623, 1597, 1513, 1494, 1284, 1242, 1156, 1123, 1080, 941, 898, $750,525 \mathrm{~cm}^{-1}$. HRMS (MALDI-TOF) calcd for $\mathrm{C}_{71} \mathrm{H}_{10} \mathrm{O}_{4}$ 926.0585, found 926.0577

[60]富勒烯稠合-4,5-二氢-3-呋喃甲酸苯酯(2e): 棕 黑色固体 $10.1 \mathrm{mg}$, 产率 23\%(回收 $\mathrm{C}_{60} 25.9 \mathrm{mg}$ ). m.p.> $300{ }^{\circ} \mathrm{C}$; UV-vis $\left(\mathrm{CHCl}_{3}\right) \lambda_{\max }: 258,314,426 \mathrm{~nm} ;{ }^{1} \mathrm{H}$ NMR (400 MHz, $\mathrm{CS}_{2} / \mathrm{DMSO}^{\left.-\mathrm{d}_{6}\right)} \delta: 8.66(\mathrm{~s}, 1 \mathrm{H}), 7.35$ (t, $J=7.9$ $\mathrm{Hz}, 2 \mathrm{H}), 7.19$ (t, $J=7.2 \mathrm{~Hz}, 3 \mathrm{H}) ;{ }^{13} \mathrm{C}$ NMR $(100 \mathrm{MHz}$, $\left.\mathrm{CS}_{2}-\mathrm{CDCl}_{3}\right) \delta: 161.37,158.56,150.25,148.01,147.22$ (4C), 146.86, 146.41, 146.27, 146.09, 145.97, 145.93, $145.49,145.47,145.14,145.01,144.58,144.43,144.07$, $143.22,142.75,142.72,142.66,142.40,142.37,142.27$, $142.23,141.61,141.36,139.91,139.86,137.58,135.38$, $129.37 ， 125.83 ， 121.70,110.88,105.92,71.54$; FT-IR (KBr) v: 2957, 2923, 2852, 1706, 1622, 1506, 1462, 1368, 1321, 1262, 1171, 1155, 1122, 1092, 940, 898, 797, 759, $748,692,576,525 \mathrm{~cm}^{-1}$. HRMS (MALDI-TOF) calcd for $\mathrm{C}_{6}{ }_{9} \mathrm{H}_{6} \mathrm{O}_{3} 882.0322$, found 882.0314.

[60]富勒烯稠合-4,5-二氢-3-呋喃甲酸-(4-乙氧基苯 基)酯(2f): 棕黑色固体 $5.6 \mathrm{mg}$, 产率 12\%(回收 $\mathrm{C}_{60} 30.7$ mg). m.p. $>300{ }^{\circ} \mathrm{C}$; UV-vis $\left(\mathrm{CHCl}_{3}\right) \lambda_{\max }: 257,315,427$ $\mathrm{nm} ;{ }^{1} \mathrm{H}$ NMR (400 MHz, $\left.\mathrm{CS}_{2}-\mathrm{CDCl}_{3}\right) \delta: 8.51(\mathrm{~s}, 1 \mathrm{H}), 7.10$ (dt, $J=9.0,5.9 \mathrm{~Hz}, 2 \mathrm{H}), 6.84(\mathrm{dt}, J=9.0,5.8 \mathrm{~Hz}, 2 \mathrm{H}), 3.99$ (q, $J=7.0 \mathrm{~Hz}, 2 \mathrm{H}), 1.41$ (t, $J=7.0 \mathrm{~Hz}, 3 \mathrm{H}) ;{ }^{13} \mathrm{C}$ NMR $(100$ $\left.\mathrm{MHz}, \mathrm{CS}_{2}-\mathrm{CDCl}_{3}\right) \delta: 161.73,158.32,156.44,147.88$, $147.15,147.08,146.75,146.27,146.13,145.95,145.83$, 145.79, 145.34 (aryl C), 145.00, 144.87, 144.45, 144.30, $143.94,143.46,143.13,142.85,142.61,142.58,142.52$, $142.26,142.23,142.13,142.09,141.47,141.23,139.76$, $139.71,137.44,135.23,122.27,114.77,110.80,105.75$, 
69.92, 63.59, 14.83; FT-IR (KBr) v: 2959, 2922, 2851, $1725,1623,1504,1464,1385,1327,1279,1180,1157$, $1112,1071,894,798,746,526 \mathrm{~cm}^{-1}$. HRMS (MALDITOF) calcd for $\mathrm{C}_{71} \mathrm{H}_{10} \mathrm{O}_{4} 926.0585$, found 926.0581 .

[60]富勒烯稠合-4,5-二氢-3-呋喃甲酸-(4-硝基苯基) 酯(2g): 棕黑色固体 $4.2 \mathrm{mg}$, 产率 9\%(回收 $\mathrm{C}_{60} 32.2 \mathrm{mg}$ ). m.p. $>300{ }^{\circ} \mathrm{C}$; UV-vis $\left(\mathrm{CHCl}_{3}\right) \lambda_{\max }: 256,314,426 \mathrm{~nm} ;{ }^{1} \mathrm{H}$ NMR (400 MHz, $\left.\mathrm{CS}_{2}-\mathrm{CDCl}_{3}\right) \delta: 8.61(\mathrm{~s}, 1 \mathrm{H}), 8.28(\mathrm{dt}, J=$ 9.1, $5.2 \mathrm{~Hz}, 2 \mathrm{H}), 7.46$ (dt, $J=9.1,5.2 \mathrm{~Hz}, 2 \mathrm{H}) ;{ }^{13} \mathrm{C} \mathrm{NMR}$ $\left(100 \mathrm{MHz}, \mathrm{CS}_{2}-\mathrm{CDCl}_{3}\right) \delta: 160.26,159.71,154.80,147.93$, $147.16,146.65,146.44,146.33,146.22,146.03,145.89$, $145.87,145.43,145.28,145.06,144.94,144.34,144.28$, $143.98,142.75,142.69,142.66,142.61,142.25,142.23$, $142.19,142.14,141.51,141.19,139.85,139.81,137.43$, $135.35,125.02,122.27,110.30,106.23,69.51$; FT-IR (KBr) v: 2973, 2923, 2851, 1732, 1619, 1519, 1456, 1401, 1384, 1343, 1325, 1283, 1200, 1158, 1109, 1050, 941, 881, $798,745,526 \mathrm{~cm}^{-1}$. HRMS (MALDI-TOF) calcd for $\mathrm{C}_{69} \mathrm{H}_{5} \mathrm{NO}_{5} 927.0173$, found 927.0172 .

[60]富勒烯稠合-4,5-二氢-3-呋喃磷酸二乙酯 $(\mathbf{2 h})$ : 棕黑色固体 $4.5 \mathrm{mg}$, 产率 10\%(回收 $\mathrm{C}_{60} 31.8 \mathrm{mg}$ ). m.p.> $300{ }^{\circ} \mathrm{C}$; UV-vis $\left(\mathrm{CHCl}_{3}\right) \lambda_{\text {max }}: 259,317,418 ;{ }^{1} \mathrm{H}$ NMR $(400$ MHz, o-Dichlorobenzene- $\left.d_{4}\right) \delta: 7.92(\mathrm{~s}, 1 \mathrm{H}), 4.10 \sim 3.94$ (m, 4H), 1.02 (t, $J=7.0 \mathrm{~Hz}, 6 \mathrm{H}) ;{ }^{13} \mathrm{C}$ NMR $(100 \mathrm{MHz}$, $o$-Dichlorobenzene- $\left.d_{4}\right) \delta: 160.62,160.36,149.48,148.87$, $148.20,147.16,147.07,146.96,146.83,146.81,146.80$, $146.36,146.17,146.03,145.88,145.59,145.21,144.97$, $144.38,143.66,143.60,143.50,143.41,143.10,143.08$ (4C), 142.52, 142.41, 140.89, 140.70, 138.24, 136.03, 119.20, 106.32, 73.03, 63.29, 63.24, 17.39, 17.32; FT-IR (KBr) $v$ : 2957, 2924, 2853, 1614, 1429, 1400, 1385, 1260, 1136, 1095, 1049, 1022, 615, 576, $527 \mathrm{~cm}^{-1}$. HRMS (MALDI-TOF) calcd for $\mathrm{C}_{66} \mathrm{H}_{11} \mathrm{O}_{4} \mathrm{P}$ 898.0400, found 898.0388.

[60]富勒烯稠合-4,5-二氢-3-呋喃苯基砜(2i): 棕黑 色固体 $14.4 \mathrm{mg}$, 产率 32\%(回收 $\mathrm{C}_{60} 23.1 \mathrm{mg}$ ). m.p.> $300{ }^{\circ} \mathrm{C}$; UV-vis $\left(\mathrm{CHCl}_{3}\right) \lambda_{\text {max }}: 255,316,420 \mathrm{~nm} ;{ }^{1} \mathrm{H}$ NMR $\left(400 \mathrm{MHz}, \mathrm{CS}_{2}-\mathrm{CDCl}_{3}\right) \delta: 8.37(\mathrm{~s}, 1 \mathrm{H}), 8.02(\mathrm{~d}, J=7.6 \mathrm{~Hz}$, $2 \mathrm{H}), 7.56(\mathrm{t}, J=7.3 \mathrm{~Hz}, 1 \mathrm{H}), 7.48(\mathrm{t}, \quad J=7.6 \mathrm{~Hz}, 2 \mathrm{H}) ;{ }^{13} \mathrm{C}$ NMR (100 MHz, $\left.\mathrm{CS}_{2}-\mathrm{CDCl}_{3}\right) \delta: 157.05,148.00,147.30$, $146.32,146.24,146.10,145.97$ (4C), 145.92, 145.52, $145.38,145.31,145.17,145.02,144.30,144.24,144.03$, $142.79,142.73,142.70,142.68,142.34,142.27,142.23$ (4C), 141.64, 141.20, 140.37 (aryl C), 139.89, 139.75, 137.36, 135.04, 133.42 (aryl), 128.97 (aryl), 128.41(aryl),
120.91, 107.14, 69.41; FT-IR (KBr) v: 2955, 2924, 2852, $1607,1513,1463,1445,1320,1184,1170,1151,1132$, 1095, 1076, 913, 887, 723, 685, 621, 606, 595, 553, 526 $\mathrm{cm}^{-1}$. HRMS (MALDI-TOF) calcd for $\mathrm{C}_{68} \mathrm{H}_{6} \mathrm{O}_{3} \mathrm{~S}$ 902.0043, found 902.0048 .

[60]富勒烯稠合-4,5-二氢-3-呋喃甲基砜(2j): 棕黑 色固体 $5.9 \mathrm{mg}$, 产率 $14 \%$ (回收 $\mathrm{C}_{60} 29.5 \mathrm{mg}$ ). m.p.> $300{ }^{\circ} \mathrm{C}$; UV-vis $\left(\mathrm{CHCl}_{3}\right) \lambda_{\max }: 257,318,422 \mathrm{~nm} ;{ }^{1} \mathrm{H}$ NMR (400 MHz, o-Dichlorobenzene- $\left.d_{4}\right) \delta: 8.01(\mathrm{~s}, 1 \mathrm{H}), 3.02(\mathrm{~s}$, $3 \mathrm{H}) ;{ }^{13} \mathrm{C}$ NMR (100 MHz, o-Dichlo- robenzene- $d_{4}$ with $\mathrm{Cr}(\mathrm{acac})_{3}$ as relaxation reagent) $\delta: 159.20,148.90,148.21$, $147.19,147.09,147.04,147.00,146.85$ (4C), 146.39, $146.37,146.18,146.03,145.93,145.10,145.06,144.96$, 143.63 (4C), 143.56, 143.42, 143.20, 143.15, 143.11, $143.02,142.51,142.17,141.09,140.77,138.26,136.53$, 122.02, 108.41, 70.77, 46.28; FT-IR (KBr) v: 3080, 2922, 2851, 2330, 1611, 1461, 1429, 1307, 1171, 1147, 987, 952, 914, 883, 797, 789, 757, 693, 576, 552, 553, $526 \mathrm{~cm}^{-1}$. HRMS (MALDI-TOF) calcd for $\mathrm{C}_{63} \mathrm{H}_{4} \mathrm{O}_{3} \mathrm{~S}$ 839.9887, found 839.9875 .

[60]富勒烯稠合-4,5-二氢-3-氰基呋喃(2k): 棕黑色 固体 $14.2 \mathrm{mg}$, 产率 36\%(回收 $\mathrm{C}_{60} 21.1 \mathrm{mg}$ ). m.p.> $300{ }^{\circ} \mathrm{C}$; UV-vis $\left(\mathrm{CHCl}_{3}\right) \lambda_{\max }: 259,316,405 \mathrm{~nm} ;{ }^{1} \mathrm{H}$ NMR $\left(400 \mathrm{MHz}, o\right.$-Dichlorobenzene- $\left.d_{4}\right) \delta: 7.76(\mathrm{~s}, 1 \mathrm{H}) ;{ }^{13} \mathrm{C}$ NMR $\left(100 \mathrm{MHz}, o\right.$-Dichlorobenzene- $\left.d_{4}\right) \quad \delta$ : 159.14, $148.98,148.24,147.20,147.15,147.00,146.86,146.82$, $146.67,146.47,146.07,146.00,145.92,145.59,145.14$, $145.05,144.94,143.63,143.54,143.52,143.18,143.07$, $143.00,142.91,142.76,142.57,142.26,141.45,140.67$, 138.24, 136.89, 114.47, 105.78, 95.32, 71.67; FT-IR (KBr) v: 2954, 2923, 2851, 1621, 1509, 1463, 1378, 1157, 891, 604, 577, $525 \mathrm{~cm}^{-1}$. HRMS (MALDI-TOF) calcd for $\mathrm{C}_{63} \mathrm{HON} 787.0064$, found 787.0059.

[60] 富勒烯稠合-4,5-二氢-2-甲基-3-呋喃甲酸乙酯

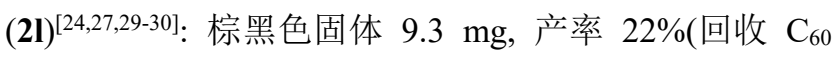
$26.0 \mathrm{mg})$. m.p. $>300{ }^{\circ} \mathrm{C}$; UV-vis $\left(\mathrm{CHCl}_{3}\right) \lambda_{\max }: 257,315$, $429 \mathrm{~nm} ;{ }^{1} \mathrm{H}$ NMR (400 MHz, $o$-Dichlorobenzene- $d_{4}$ ) $\delta$ : 4.08 (q, $J=7.1 \mathrm{~Hz}, 2 \mathrm{H}), 2.65$ (s, 3H), 0.998 (t, $J=7.1 \mathrm{~Hz}$, $3 \mathrm{H}) ;{ }^{13} \mathrm{C}$ NMR $\left(100 \mathrm{MHz}, \mathrm{CS}_{2}-\mathrm{CDCl}_{3}\right) \delta: 168.71,163.50$, $148.14,147.63,146.98,146.91,146.05,145.83,145.76$, $145.62,145.57,145.26,145.00,144.79,144.63,144.38$, $144.14,144.03,143.84,142.43,142.39,142.31,142.18$, $142.07,141.95,141.90,141.19,141.07,139.51,139.00$, 137.07, 134.89, 104.59, 102.33, 71.78, 60.19, 15.24, 14.18 . FT-IR (KBr) v: 2954, 2922, 2852, 1701, 1625, 1508, 1462, 
1371, 1323, 1300, 1172, 1155, 1126, 1098, 975, 797, 760, $573,526 \mathrm{~cm}^{-1}$. HRMS (MALDI-TOF) calcd for $\mathrm{C}_{66} \mathrm{H}_{8} \mathrm{O}_{3}$ 848.0479 , found 848.0458.

\subsection{1 对照实验中 21 的合成}

根据文献[29]报道的合成方法，在装有摚拌子的封 管中加入 $36.0 \mathrm{mg} \mathrm{C}_{60}, 65 \mu \mathrm{L}$ 乙酰乙酸乙酯(10 equiv.), $20.1 \mathrm{mg} \mathrm{Cu}(\mathrm{OAc})_{2}$ (2 equiv.) 和 $30.5 \mathrm{mg}$ DMAP (5 equiv.), 并加入 $7 \mathrm{~mL}$ 邻二氯苯. 该反应混合物在 $80{ }^{\circ} \mathrm{C}$ 的油浴下 反应 $1 \mathrm{~h}$, 停止反应. 减压蒸馏除去邻二氯苯, 用二硫化 碳溶解后进行柱色谱分离, 得到 $12.6 \mathrm{mg}$ (产率 30\%)的 产物 2l ${ }^{[24,27,29-30]}$. ${ }^{1} \mathrm{H}$ NMR (400 MHz, $o$-Dichlorobenzene$\left.d_{4}\right) \delta: 4.08(\mathrm{q}, J=7.1 \mathrm{~Hz}, 2 \mathrm{H}), 2.65(\mathrm{~s}, 3 \mathrm{H}), 0.998(\mathrm{t}, J=$ $7.1 \mathrm{~Hz}, 3 \mathrm{H}) ;{ }^{13} \mathrm{C}$ NMR $\left(100 \mathrm{MHz}, \mathrm{CS}_{2}-\mathrm{CDCl}_{3}\right) \delta: 168.71$, $163.64,148.04,147.55,146.86,146.83,145.97,145.75$, $145.68,145.54,145.48,145.18,144.92,144.71,144.54$, $144.28,144.06,143.93,143.76,142.34,142.31,142.23$, $142.09,141.98,141.86,141.82,141.11,140.98,139.43$, $138.93,137.00,134.82,104.52,102.28,71.69,60.12$, $15.14,14.02$.

辅助材料(Supporting Information) 化合物 $\mathbf{2 a} \sim 21$ 的 ${ }^{1} \mathrm{H} N M R,{ }^{13} \mathrm{C} \mathrm{NMR}$ 和 HRMS 谱图. 这些材料可以免费 从本刊网站(http://sioc-journal.cn/)上下载.

\section{References}

[1] Gaspar, H.; Figueira, F.; Pereira, L.; Mendes, A.; Viana, J. C.; Bernardo, G. Materials 2018, 11, 2560.

[2] Akiyama, T. Bull. Chem. Soc. Jpn. 2019, 92, 1181.

[3] Umeyama, T.; Imahori, H. Acc. Chem. Res. 2019, 52, 2046.

[4] Speller, E. M.; Clarke, A. J.; Luke, J.; Lee, H. K. H.; Durrant, J. R.; Li, N.; Wang, T.; Wong, H. C.; Kim, J.-S.; Tsoi, W. C.; Li, Z. J. Mater. Chem. A 2019, 7, 23361.

[5] Wan, X.; Li, C.; Zhang, M.; Chen, Y. Chem. Soc. Rev. 2020, 49, 2828.

[6] Cheng, P.; Shi, Q.; Zhan, X. Acta Chim. Sinica 2015, 73, 252 (in Chinese). (程沛, 史钦钦, 占肖卫, 化学学报, 2015, 73, 252.)

[7] Sun, Y.; Gao, H.; Zhang, Y.; Wang, Y.; Kan, B.; Wan, X.; Zhang, H.; Chen, Y. Chin. J. Org. Chem. 2018, 38, 228 (in Chinese). (孙延娜, 高欢欢, 张雅敏, 王云闯, 阚斌, 万相见, 张洪涛, 陈 永胜, 有机化学, 2018, 38, 228.)

[8] Yao, S.; Yuan, X.; Jiang, L.; Xiong, T.; Zhang, J. Materials 2020, 13, 2924.

[9] Pan, Y.; Liu, X.; Zhang, W.; Liu, Z.; Zeng, G.; Shao, B.; Liang, Q.; He, Q.; Yuan, X.; Huang, D.; Chen, M. Appl. Catal. B: Environ. 2020, 265, 118579.

[10] Krokosz, A.; Lichota, A.; Nowak, K. E.; Grebowski, J. Radiat. Phys. Chem. 2016, 128, 143 .

[11] Ghasemzadeh, P.; Rezayat, S. M.; Adeli, S.; Rahbar-Roshandel, N. Acta Med. Iran. 2016, 54, 478.

[12] Goodarzi, S.; Da Ros, T.; Conde, J.; Sefat, F.; Mozafari, M. Mater. Today 2017, 20, 460 .
[13] Rašović, I. Mater. Sci. Technol. 2017, 33, 777.

[14] Pochkaeva, E. I.; Podolsky, N. E.; Zakusilo, D. N.; Petrov, A. V.; Charykov, N. A.; Vlasov, T. D.; Penkova, A. V.; Vasina, L. V.; Murin, I. V.; Sharoyko, V. V.; Semenov, K. N. Prog. Solid State Chem. 2020, 57, 100255 .

[15] Hirsch, A.; Brettreich, M. Fullerenes: Chemistry and Reactions, Wliey-VCH, Weinheim, Germany, 2005.

[16] Kadish, K. M.; Ruoff, R. S. Fullerenes: Chemistry, Physics, and Technology, Wiley, New York, 2000.

[17] Martín, N. Chem. Commun. 2006, 2093.

[18] Shi, L.; Wang, C.; Xu, Y.; Wang, Q. Chin. J. Org. Chem. 2015, 35, 1537 (in Chinese). (史玲, 王彩芳, 徐雨, 汪秋安, 有机化学, 2015, 35, 1537.)

[19] Chen, S.; Liu, H.; Liu, Z.; Li, S.; Chen, Y.; Li, H.; Li, D.; Zhang, W. Chin. J. Org. Chem. 2020, 40, 209 (in Chinese). (陈书帅, 刘洪新, 刘昭明, 李赛妮, 陈玉婵, 李浩华, 李冬利, 章卫民，有机化学, 2020, 40, 209.)

[20] Kornsakulkarn, J.; Saepua, S.; Srichomthong, K.; Supothina, S.; Thongpanchang, C. Tetrahedron 2012, 68, 8480.

[21] Li, S.; Hu, J.; Zhang, L.; Zhang, L.; Sun, Y.; Xie, Y.; Wu, S.; Liu, L.; Gao, Z. J. Pharm. Pharmacol. 2013, 65, 355.

[22] Husain, A.; Khan, S. A.; Iram, F.; Iqbal, M. A.; Asif, M. Eur. J. Med. Chem. 2019, 171, 66.

[23] Lien, J.-C.; Lin, C.-S.; Lai, H.-C.; Tsai, Y.-C.; Lin, Y.-F.; Huang, A.-C.; Huang, S.-H.; Lin, C.-W. Bioorg. Med. Chem. Lett. 2019, 29, 126742 .

[24] Ohno, M.; Yashiro, A.; Eguchi, S. Chem. Commun. 1996, 291.

[25] Chen, S.; Li, Z.-J.; Gao, X. J. Org. Chem. 2016, 81, 121.

[26] Yang, H.-L.; Xu, L.-J.; Li, W.-Z.; Sun, T.; Duan, B.-R.; Chen, S.; Gao, X. RSC Adv. 2020, 10, 24549

[27] Zhang, T.-H.; Wang, G.-W.; Lu, P.; Li, Y.-J.; Peng, R.-F.; Liu, Y.-C.; Murata, Y.; Komatsu, K. Org. Biomol. Chem. 2004, 2, 1698.

[28] Cheng, X.; Wang, G. W.; Murata, Y.; Komatsu, K. Chin. Chem. Lett. 2005, 16, 1327.

[29] Wang, G.-W.; Li, F.-B. Org. Biomol. Chem. 2005, 3, 794.

[30] Li, F.; Zhu, S.; You, X.; Wang, G. Chin. Sci. Bull. 2012, 57, 2269.

[31] Li, C.; Zhang, D.; Zhang, X.; Wu, S.; Gao, X. Org. Biomol. Chem. 2004, 2, 3464.

[32] Xia, S.; Liu, T.-X.; Zhang, P.; Ma, J.; Liu, Q.; Ma, N.; Zhang, Z.; Zhang, G. J. Org. Chem. 2018, 83, 862.

[33] Wu, X. F.; Neumann, H.; Beller, M. Chem. Rev. 2013, 113, 1.

[34] Li, J.; Yang, S.; Wu, W.; Jiang, H. Org. Chem. Front. 2020, 7, 1395.

[35] Li, J.; Yang, S.; Wu, W.; Jiang, H. Chem. Asian J. 2019, 14, 4114.

[36] Ma, X.; Yu, J.; Wang, Z.; Zhang, Y.; Zhou, Q. Chin. J. Org. Chem. 2020, 40, 2669 (in Chinese)

(马献涛, 于静, 王子龙, 张枅, 周秋菊, 有机化学, 2020, 40, 2669.)

[37] Zhu, B.; Wang, G.-W. Org. Lett. 2009, 11, 4334

[38] Su, Y.-T.; Wang, Y.-L.; Wang, G.-W. Org. Chem. Front. 2014, 1, 689.

[39] Hussain, M.; Niu, C.; Wang, G.-W. Org. Chem. Front. 2020, 7 , 1249.

[40] Wang, C.; Liu, Z.; Yin, Z.-C.; Wang, G.-W. Org. Chem. Front. 2020, 7, 2518.

[41] Li, F.; Wang, J.-J.; Wang, G.-W. Chem. Commun. 2017, 53, 1852.

[42] Yan, Y.-T.; Gao, W.; Jin, B.; Shan, D.-S.; Peng, R. F.; Chu, S.-J. J. Org. Chem. 2018, 83, 672

[43] Dai, C.; Han, Y.; Liu, L.; Huang, Z.-B.; Shi, D.-Q.; Zhao, Y. Org. Chem. Front. 2020, 7, 1703.

[44] Zhang, J.; Han, L.; Bi, S.; Liu, T. J. Org. Chem. 2020, 85, 8387.

[45] Kumar, P.; Kapur, M. Org. Lett. 2019, 21, 2134.

[46] Wright, J. A.; Gaunt, M. J.; Spencer, J. B. Chem. Eur. J. 2006, 12, 949. 